\title{
ANÁLISE DA CARBONATAÇ̃̃o E VIDA ÚTIL DE PROJETO DE ESTRUTURAS DE CONCRETO VIA SIMULAÇÃO DE MONTE CARLO
}

\author{
PEIXOTO, DAIANE DA SILVA \\ Graduanda em Eng. Civil \\ Centro Universitário Estácio de Ribeirão Preto \\ Ribeirão Preto/SP; Brasil \\ daianesilvapeixoto@hotmail.com
}

\author{
FELIX, EMERSON FELIPE \\ Doutorando em Eng. Civil/Estruturas \\ Departamento de estruturas EESC/USP \\ São Carlos/SP; Brasil \\ emerson.felipe.felix@gmail.com
}

\section{RESUMO}

O presente trabalho apresenta uma análise numérico-probabilística da despassivação do aço ao longo da vida útil de projeto de estruturas de concreto armado situadas em diferentes regiões do Brasil. A determinação da profundidade de carbonatação foi realizada considerando a não linearidade dos parâmetros envolvidos no fenômeno. A probabilidade de despassivação foi calculada por meio de simulações de Monte Carlo. Os resultados encontrados apontam que a depender da resistência à compressão, do cobrimento e do ambiente de exposição, a carbonatação tem seu processo de desenvolvimento acelerado, e que a probabilidade de o concreto de cobrimento proteger o aço da corrosão ao longo da sua vida útil de projeto é variável para cada região (sul, sudeste, norte, nordeste ou centro-oeste), sendo dependente das condições climáticas de cada local, demonstrando a importância da consideração da aleatoriedade dos parâmetros do fenômeno para estudos de vida útil.

Palavras-chave: Concreto armado, carbonatação do concreto, despassivação do aço, simulação de Monte Carlo.

\section{ABSTRACT}

This paper presents a numerical probabilistic analysis of the depassivation of steel over the design life of reinforced concrete structures located in different regions of Brazil. The carbonation depth was determined considering the nonlinearity of the parameters involved in the phenomenon. The probability of depassivation was calculated by Monte Carlo simulations. The results show that, depending on the compressive strength, the concrete covering and the exposure environment, carbonation has its development accelerated, and that the probability of the concrete coatings protect steel from corrosion throughout its design life varies for each region (south, southeast, north, northeast, or midwest), being dependent on the climatic conditions of each location, demonstrating the importance of considering the randomness of the parameters of the phenomenon for useful life studies.

Keywords: reinforced concrete, concrete carbonation, steel depassivation, Monte Carlo simulation.

\section{INTRODUÇÃO}

Uma das principais causas de redução da vida útil de estruturas de concreto armado é a corrosão, uma vez que esta envolve a perda de material da superfície do aço como resultado de uma ação química, e a perda material leva a redução de área efetiva na seção transversal e consequentemente a diminuição da capacidade de suportar cargas (KARI et al., 2014). No Brasil, por exemplo, a corrosão possuí um índice de ocorrência em estruturas de concreto que varia de 14 a $64 \%$ a depender da região de análise (CARMONA; MAREGA, 1988; DAL MOLIN, 1988; ANDRADE, 1992).

A corrosão das armaduras de aço no concreto é um processo eletroquímico, causado pelas diferenças nas concentrações de íons dissolvidos, de maneira que parte do metal se torna catódica e outra anódica, resultando na perda de volume do material, gerando produtos de corrosão, e consequente redução de capacidade de suporte (MEHTA; MONTEIRO, 2014; GEIKER; POLDER, 2016). A formação dos produtos de corrosão gera um campo de tensões interno, localizado na zona de interface entre o aço e o concreto, que em função da sua magnitude leva a formação de fissuras que se propagam em direção às faces de concreto expostas à atmosfera (KARI et al., 2014; NGUYEN et al., 2015). 
De acordo com Helene (2018) e a corrosão está, em geral, associada à carbonatação do concreto (corrosão uniforme) ou ao ingresso de cloretos (corrosão localizada). No que condiz o processo corrosivo iniciado pela carbonatação do concreto, o processo pode ser subdividido em duas fases, a iniciação (Figura 1a) e a propagação (Figura 1c), sendo a transição entre as duas fases caracterizada pela despassivação do aço (Figura 1b), momento em que a profundidade de difusão do $\mathrm{CO}_{2}$ $\left(\mathrm{d}_{\mathrm{d}}\right)$ é igual ao cobrimento da armadura $\left(\mathrm{c}_{1}\right)$.

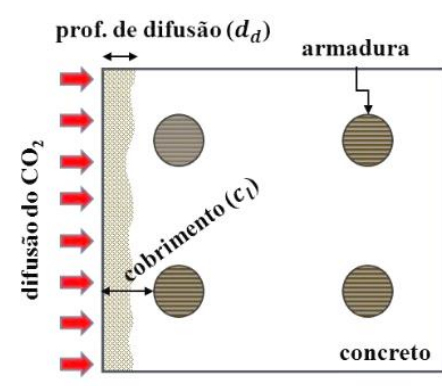

(I) Iniciação $\left(d_{d}<c_{l}\right)$

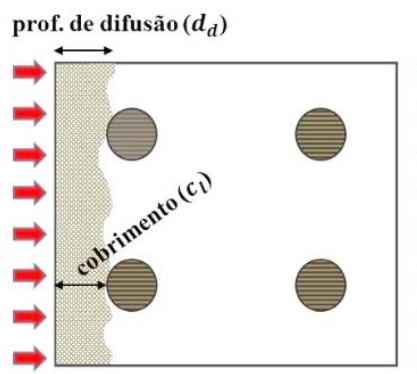

(II) Despassivação $\left(d_{d}=c_{l}\right)$

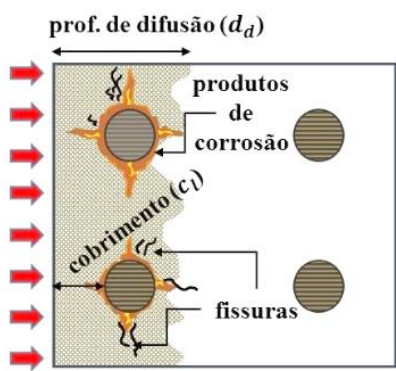

(III) Propagação $\left(d_{d}>c_{l}\right)$

Figura 1: Estágios do processo de corrosão por carbonatação (FELIX et al., 2019)

A fase de iniciação (Figura 1a) se caracteriza pela difusão de diferentes agentes agressivos como o dióxido de carbono $\left(\mathrm{CO}_{2}\right)$ presentes no meio ambiente. $\mathrm{O}$ gás carbônico penetra na matriz porosa do concreto reagindo com os hidróxidos de cálcio $\left(\mathrm{Ca}(\mathrm{OH})_{2}\right)$ da pasta de cimento, levando a formação do carbonato de cálcio $\left(\mathrm{CaCO}_{3}\right)$. Este processo de carbonatação é responsável pela redução da porosidade e permeabilidade (NEVILLE, 1997). Além disso, a carbonatação ocasiona na redução do pH do concreto (de 13 a aproximadamente 8), e consequentemente, na destruição da camada passivadora que envolve a armadura, deixando-a suscetível à corrosão (CHANG; CHEN, 2006).

À medida que a frente de carbonatação atinge a profundidade do aço e promove sua despassivação, tem-se início a fase de propagação da corrosão. Até o instante de despassivação do aço, os danos não são perceptíveis, diferentemente do que ocorre durante o período de propagação (TUUTTI, 1982; POSSAN, 2010; KÖLIÖ et al., 2017; FELIX et al., 2019).

No que condiz a modelagem destes processos, boa parte das pesquisas se concentram no estudo do estágio de iniciação da corrosão, com o desenvolvimento de modelos para a estimativa da frente de carbonatação. Os primeiros modelos para a previsão da profundidade de carbonatação foram obtidos por meio de regressões linear e não linear, considerando diferentes fatores, por exemplo, a relação água/cimento (a/c), o tipo do aglomerante e as condições de exposição, como é visto nos trabalhos de Hamada (1968), Smolczyk (1968) e Helene (2018).

Entretanto, um complicador natural da avaliação da corrosão é dado pela difusão do $\mathrm{CO}_{2}$ na matriz porosa do concreto. A difusão é controlada por complexas interações entre os mecanismos físicos e químicos dos materiais, onde várias fontes de incertezas podem ser observadas (MEHTA; MONTEIRO, 2014). A penetração do gás carbônico é muitas vezes simplificada, sem perda significativa de generalidade, por problemas controlados apenas pelo processo de difusão. Além disso, existem as incertezas presentes nas propriedades dos materiais, nas condições ambientais e nos próprios parâmetros dos modelos matemáticos que simulam a corrosão, dificultando ainda mais as análises.

Diante do exposto, abordagens puramente determinísticas não são capazes de avaliar adequadamente a vida útil de estruturas em concreto armado sujeitas ao processo de corrosão, sendo necessário analisar o problema considerando a aleatoriedade dos diferentes parâmetros. Assim, neste trabalho analisa-se a probabilidade de um elemento de concreto armado ter suas armaduras despassivadas durante a vida útil de projeto (50 anos) a partir de uma abordagem combinada entre a teoria da confiabilidade e formulações matemáticas referentes aos processos de carbonatação e despassivação do aço.

\section{MODELAGEM PROBABILISTICA DA VIDA ÚTIL DE PROJETO}

A fim de determinar a probabilidade de despassivação do aço em vigas de concreto armado em um período de 50 anos, período descrito como vida útil de projeto, de acordo com a NBR 6118 (ABNT, 2014), uma abordagem mecânicaprobabilística via simulação de Monte Carlo foi considerada neste trabalho, de forma a inserir no processo de modelagem a aleatoriedade dos parâmetros envolvidos no fenômeno. Assim, na sequência são apresentadas as formulações analíticas utilizadas para modelar o processo de difusão do $\mathrm{CO}_{2}$ no concreto, e estimar o tempo de despassivação do aço devido a 
carbonatação do concreto. São apresentadas ainda, o acoplamento destas formulações à metodologia de análise probabilística.

\subsection{Levantamento de dados e análise das variáveis aleatórias do problema}

Para a análise probabilística via simulação de Monte Carlo, faz-se necessário descrever as variáveis aleatórias do problema de estudo e suas funções de distribuição. Para tal, inicialmente foram realizadas coletas de dados acerca da umidade relativa do ar ao longo dos últimos 10 anos, assim como a concentração de $\mathrm{CO}_{2}$ na atmosfera.

Os dados foram coletados para cinco capitais do território brasileiro, sendo: Manaus (AM); Fortaleza (CE); Brasília (DF); São Paulo (SP); Florianópolis (SC). As cinco cidades foram selecionadas de modo a pegar uma capital para cada uma das regiões do brasil (Sul, Sudeste, Norte, Nordeste, Centro-Oeste). Na Figura 2 são apresentadas as cidades selecionadas para a análise probabilística da vida útil de projeto, com as respectivas frequências de distribuição das umidades coletadas do banco de dados meteorológicos para ensino e pesquisa (BDMEP) do Instituto Nacional de Meteorologia (INMET).

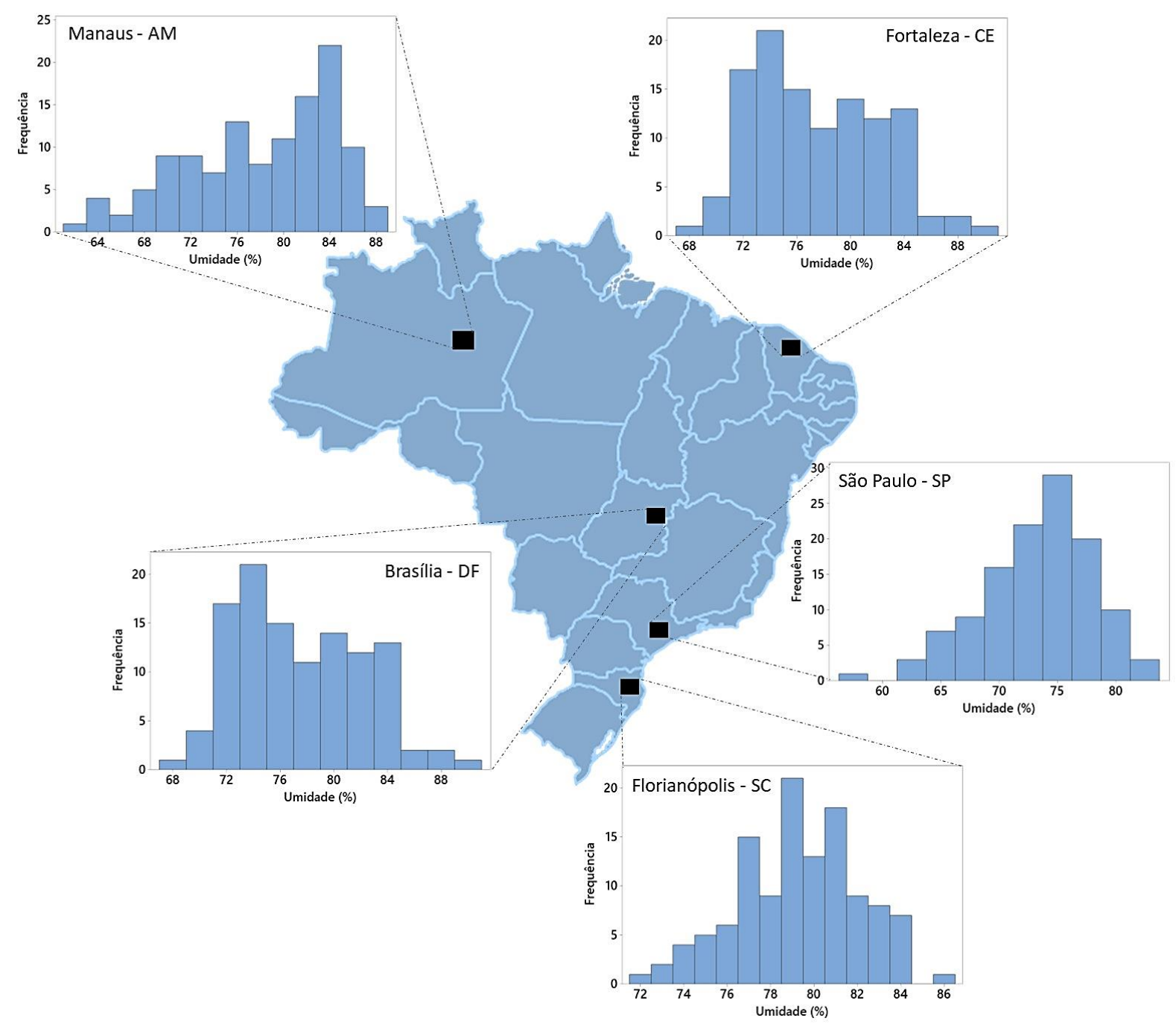

Figura 2: Cidades analisadas e distribuição das frequências das umidades.

Além das médias e dos desvios, para a realização das análises probabilísticas, faz-se necessário determinar as funções de distribuição que melhor representam as variáveis aleatórias, assim, na Figura 3, são apresentadas as funções de distribuições adotadas para a representação da umidade relativa do ar de cada cidade. Comenta-se que para as cidades de Brasília, Manaus e Fortaleza, foram realizadas transformações de Johnson para então descrever a umidade com uma 
função de distribuição normal. Para Florianópolis, observou-se que os dados se adequavam em uma distribuição normal, enquanto para São Paulo, uma distribuição de Weibull foi adotada.
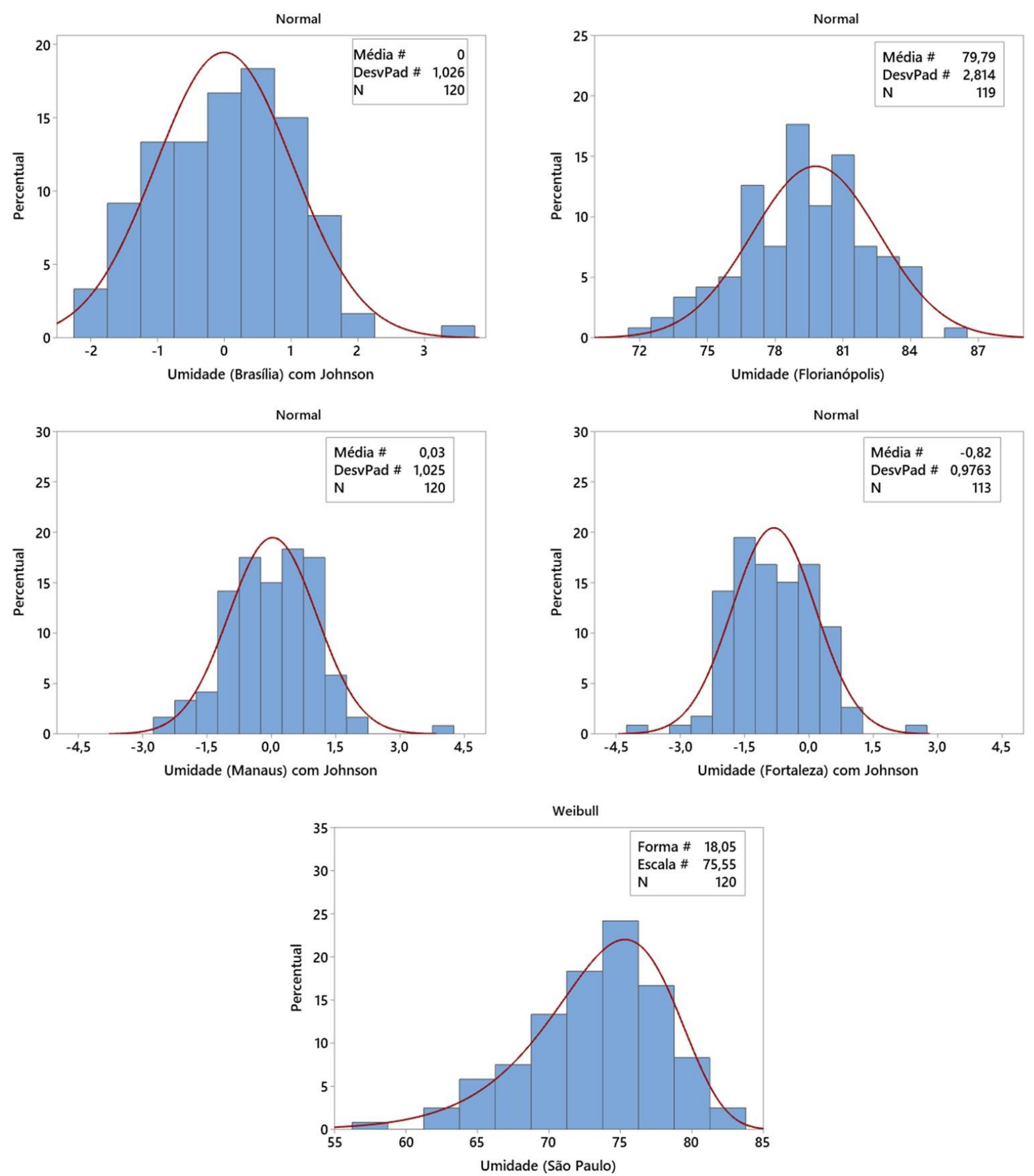

Figura 3: Funções de distribuição.

Para a determinação da média, desvio e função de distribuição da concentração de $\mathrm{CO}_{2}$ na atmosfera, não foi possível coletar dados para cada uma das cidades, por falta de informações disponíveis em agencias de mensuração por órgãos governamentais. Assim, decidiu-se utilizar dados globais, coletados do órgão $\mathrm{CO}_{2}$ Levels.

\subsection{Descrição do modelo implementado para análise da despassivação do aço}

Para a determinação da profundidade de carbonatação ao longo do tempo, e consequentemente, do tempo de despassivação do aço, foi utilizada a formulação analítica proposta por Possan (2010), que é apresentada na Equação 1. . Alguns dos parâmetros da formulação são obtidos em função das características do concreto analisado e do ambiente ao qual está inserido, outros parâmetros são coletados das Tabelas 1.a e 1.b. 


$$
y=k_{c}\left(\frac{20}{f_{c}}\right)^{k_{f c}} \cdot\left(\frac{t}{20}\right)^{\frac{1}{2}} \cdot \exp \left[\left(\frac{k_{a d} \cdot a d^{\frac{3}{2}}}{40+f_{c}}\right)+\left(\frac{k_{c o_{2}} \cdot C O_{2}}{60+f_{c}}\right)-\left(\frac{k_{R U} \cdot(U R-0,58)^{2}}{100+f_{c}}\right)\right] \cdot k_{c e}
$$

Onde:

$\mathrm{y}=$ profundidade de carbonatação média do concreto, em $\mathrm{mm}$;

$\mathrm{f}_{\mathrm{c}}=$ resistência característica à compressão axial do concreto, em $\mathrm{MPa}$;

$\mathrm{k}_{\mathrm{c}}=$ fator variável referente ao tipo de cimento empregado (Tabela 1.a);

$\mathrm{k}_{\mathrm{fc}}=$ fator variável referente à resistência à compressão axial do concreto, em função do tipo de cimento utilizado (Tabela

1.a);

$\mathrm{t}=$ idade do concreto, em anos;

$\mathrm{a}_{\mathrm{d}}=$ teor de adição pozolânica no concreto, em \% em relação à massa de cimento;

$\mathrm{k}_{\mathrm{ad}}=$ fator variável referente às adições pozolânicas do concreto - sílica ativa, metacaulim e cinza de casca de arroz, em função do tipo de cimento utilizado (Tabela 1.a).

$\mathrm{UR}=$ umidade relativa média, em $\% * 0,01$;

$\mathrm{K}_{\mathrm{RU}}=$ fator variável referente à umidade relativa, em função do tipo de cimento utilizado (Tabela 1.a)

$\mathrm{CO}_{2}=$ teor de $\mathrm{CO}_{2}$ da atmosfera, em $\%$.

$\mathrm{k}_{\mathrm{co} 2}$ = fator variável referente ao teor de $\mathrm{CO}_{2}$ do ambiente, em função do tipo de cimento utilizado (Tabela 1.a)

$\mathrm{k}_{\mathrm{ce}}=$ fator variável referente à exposição à chuva, em função das condições de exposição da estrutura (Tabela 1.b).

Tabela 1- Coeficientes do modelo em função (a) das características do concreto e das condições ambientais (b) das condições de exposição (POSSAN, 2010).

a)

\begin{tabular}{c|c|c|c|c|c}
\hline \multirow{2}{*}{$\begin{array}{c}\text { Tipo de } \\
\text { Cimento }\end{array}$} & \multicolumn{2}{|c|}{ Características do concreto } & \multicolumn{2}{c}{$\begin{array}{c}\text { Condições } \\
\text { Ambientais }\end{array}$} \\
\cline { 2 - 6 } & Cimento & $\mathrm{f}_{\mathrm{c}}$ & Adição & $\mathrm{CO}_{2}$ & $\mathrm{UR}$ \\
\cline { 2 - 6 } & $\mathrm{k}_{\mathrm{c}}$ & $\mathrm{k}_{\mathrm{fc}}$ & $\mathrm{k}_{\mathrm{ad}}$ & $\mathrm{k}_{\mathrm{CO} 2}$ & $\mathrm{k}_{\mathrm{UR}}$ \\
\hline CP I & 19,80 & 1,70 & 0,24 & 18,00 & 1300 \\
\hline CP II E & 22,48 & 1,50 & 0,32 & 15,50 & 1300 \\
\hline CP II F & 21,68 & 1,50 & 0,24 & 18,00 & 1100 \\
\hline CP II Z & 23,66 & 1,50 & 0,32 & 15,50 & 1300 \\
\hline CP III & 30,50 & 1,70 & 0,32 & 15,50 & 1300 \\
\hline CP IV & 33,27 & 1,70 & 0,32 & 15,50 & 1000 \\
\hline CP V ARI & 19,80 & 1,70 & 0,24 & 18,00 & 1300 \\
\hline
\end{tabular}

b)

\begin{tabular}{l|c}
\hline \multicolumn{2}{c}{$\begin{array}{c}\text { Condições de exposição da } \\
\text { estrutura }\end{array}$} \\
\hline \multicolumn{1}{c|}{ Proteção à chuva } & $k_{c e}$ \\
\hline $\begin{array}{l}\text { Ambiente interno Protegido } \\
\text { da chuva }\end{array}$ & 1,30 \\
\hline $\begin{array}{l}\text { Ambiente externo } \\
\text { Protegido da chuva }\end{array}$ & 1,00 \\
\hline $\begin{array}{l}\text { Ambiente externo } \\
\text { Desprotegido da chuva }\end{array}$ & 0,65 \\
\hline
\end{tabular}

\subsection{Analise probabilística via simulação de Monte Carlo}

O método de simulação de Monte Carlo é um procedimento numérico, amplamente utilizado em diversas áreas, para a realização de experimentos aleatórios em problemas de confiabilidade. Neste método, amostras de variáveis aleatórias são utilizadas para construir um conjunto de realizações do experimento com o intuito de descrever a falha, os espaços seguros, correlacionados à confiabilidade, e calcular a probabilidade do evento, conforme Equação (2). As formulações aqui descritas, foram resumidas de acordo com Liberati et al. (2014).

As amostras utilizadas para o cálculo da probabilidade de falha, são construídas com base na distribuição estatística atribuída a cada variável aleatória do problema. Como o método está baseado na simulação da função de estado limite, quanto maior for a amostra gerada, mais precisa será a descrição do espaço e mais precisa será a probabilidade de falha ou confiabilidade determinada (BECK, 2018).

$$
P_{f}=\int_{G \leq 0} f_{x}\left(x_{1}, x_{2}, \ldots, x_{n}\right) d x_{1}, d x_{2}, \ldots, d x_{n}
$$

Como a avaliação da integral definida na Equação (2) é quase impossível na prática, procedimentos alternativos foram desenvolvidos com base no conceito de índice de confiabilidade $\beta$ (BECK, 2018). O índice de confiabilidade é definido como a distância entre o ponto médio e o ponto de falha alocado sobre a função de estado limite, $\mathrm{G}(\mathrm{X})=0$. 
De forma geral, o método consiste na construção de uma amostra para as variáveis aleatórias envolvidas no problema a ser simulado, e assim, a probabilidade de falha é calculada utilizando um estimador, baseado na avaliação da função de estado limite, de acordo como apresentado na Equação (3). O estimador I(xi), baseado na função de estado limite, é dado em (9), sendo seu valor 0 ou 1, sendo 0 para o caso em que não há falha e, 1, para quando há falha.

$$
\begin{gathered}
P_{f}=\int_{G \leq 0} f_{x}\left(x_{i}\right) d x_{i}=\int_{G \leq 0} I\left(x_{i}\right) f_{x}\left(x_{i}\right) d x_{i}=E\left[I\left(x_{i}\right)\right] \\
I\left(x_{i}\right)= \begin{cases}1, & G \leq 0 \\
0, & G>0\end{cases}
\end{gathered}
$$

Simulando a função de estado limite para um número conveniente de amostras, o valor médio I(xi) será uma estimativa para a probabilidade de falha, assim, de acordo com a Equação (5) a probabilidade de falha pode ser facilmente estimada para todo o conjunto de amostras.

$$
\overline{P_{f}}=E\left[I\left(x_{i}\right)\right]=\frac{1}{N} \sum_{i=1}^{N} I\left(x_{i}\right)
$$

em que N é o número de simulações, isto é, de avaliações da equação de estado limite do problema analisado.

Por fim, definido o método de estimativa da probabilidade de falha, a equação de estado limite a ser analisada neste trabalho é dada na Equação (6) e, refere-se ao evento da viga ter suas armaduras despassivadas durante a vida útil de projeto (em 50 anos).

$$
g(x)=C-y
$$

em que C é o cobrimento da armadura (em mm), e y, é a profundidade de carbonatação do concreto (em mm), calculada pela Equação (1). Os cobrimentos foram definidos em função do ambiente de exposição e da sua agressividade, conforme descrito na NBR 6118 (ABNT,2014).

De acordo com a expressão apresentada em (6), a falha do evento ocorre quando g $\leq 0$, e não falha quando g $>0$. Assim, em nossas análises, a falha ocorre quando a profundidade de carbonatação for igual ou superior cobrimento da armadura. Os demais casos representam a não falha do evento.

\subsection{Descrição das estruturas e cenários analisados}

As estruturas analisadas neste trabalho, consistem em vigas de concreto armado dimensionadas de acordo com os procedimentos da NBR 6118 (ABNT, 2014). O esquema que representa o carregamento utilizado para o dimensionamento no estado limite último (ELU) e para a análise do estado limite de serviço (ELS) são apresentados na Figura 4, assim como os detalhes construtivos da mesma.

Para a definição das vigas e as simulações, as variáveis foram caracterizadas como determinísticas ou aleatórias. As variáveis determinísticas foram divididas em variáveis com valores fixos e não fixos, sendo as variáveis com valores fixos: altura de $40 \mathrm{~cm}$; largura da seção transversal de $22 \mathrm{~cm}$; barras longitudinais com $2 \mathrm{~cm}$ de diâmetro; concretos produzidos com relação água cimento de 0,4 .

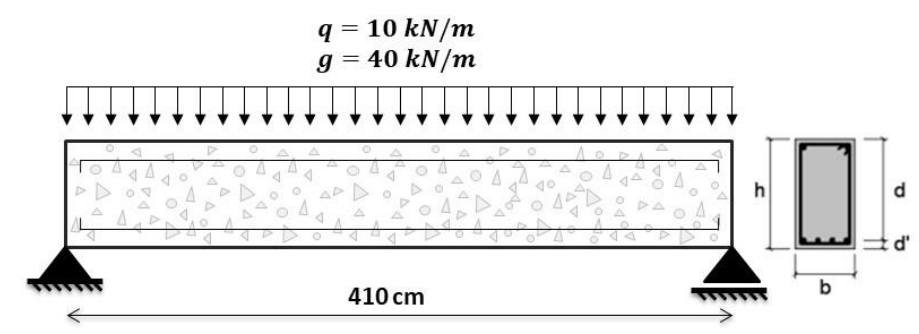

Figura 4: Condições de carregamento e geometria da viga. 
As variáveis determinísticas com valores não fixos, foram definidas com o propósito de analisar a sua influência no processo de degradação das vigas, sendo: $\mathrm{k}_{\mathrm{c}}, \mathrm{k}_{\mathrm{fc}}, \mathrm{k}_{a d}, \mathrm{k}_{\mathrm{ur}}, \mathrm{k}_{\mathrm{CO} 2}$ (dependentes do tipo de cimento, de acordo com a Tabela 1), e $\mathrm{k}_{\mathrm{ce}}$ (dependente do tipo de exposição à chuva, ver de acordo com a Tabela 2).

No que diz respeito às variáveis aleatórias, neste trabalho é analisada a variabilidade presente em quatro parâmetros relacionados à despassivação: (i) o cobrimento das armaduras; (ii) a resistência à compressão do concreto; (iii) a umidade relativa e; (iv) o teor de $\mathrm{CO}_{2}$ no ambiente. A Tabela 3 apresenta as quatro variáveis aleatórias com suas respectivas médias, coeficiente de variação e função de distribuição.

Tabela 3 - Dados referentes ao concreto e ambiente de exposição.

\begin{tabular}{c|c|c|c}
\hline Variável & Média & Coeficiente de variação & Distribuição \\
\hline Cobrimento $(\mathrm{mm})$ & 30 & $10 \%$ & Normal \\
\hline Resistência à compressão do concreto $(\mathrm{MPa})$ & 25 & $15 \%$ & Normal \\
\hline Umidade relativa $(\%)$ & \multicolumn{3}{|c}{ Depende da cidade } \\
\hline Teor de $\mathrm{CO}_{2}(\%)$ & 0,03 & $18 \%$ & Log-Normal \\
\hline
\end{tabular}

\section{RESULTADOS}

Inicialmente, na Figura 5 é apresentado o avanço da profundidade de carbonatação do concreto para as cinco cidades analisadas neste trabalho (cidades apresentadas na Figura 2), e os diferentes ambientes de exposição simulados (Ambiente interno protegido da chuva e externo desprotegido da chuva). Os resultados foram obtidos considerando os valores médios das variáveis aleatórias do problema, conforme Tabela 3 e Figura 3.
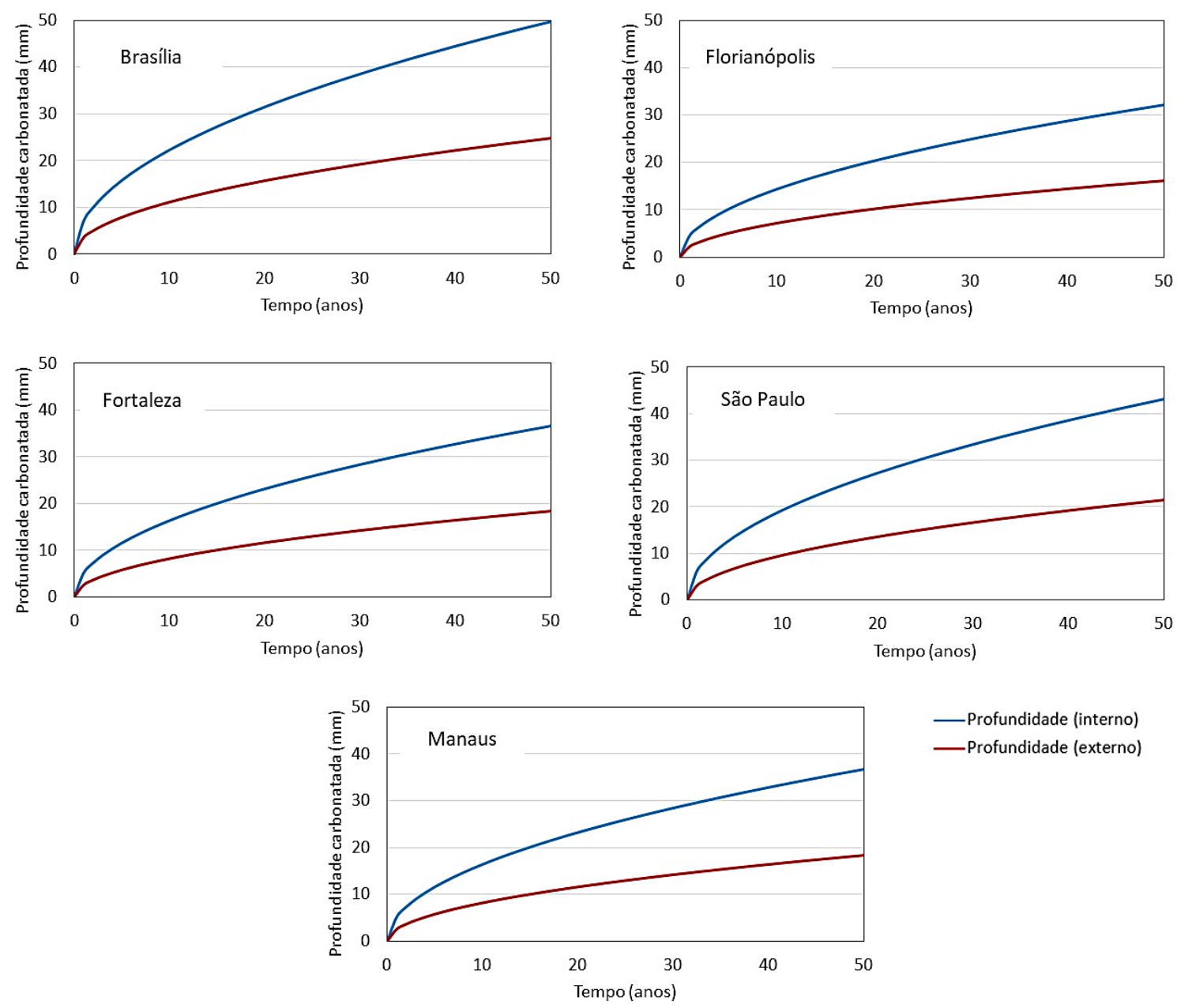

- Profundidade (interno)

- Profundidade (externo)

Figura 5: Avanço da carbonatação no concreto e área útil de aço. 
Observa-se na Figura 5 que ao considerarmos as variáveis como determinísticas, e sabendo que a média adotada para o cobrimento foi de $30 \mathrm{~mm}$, todas as estruturas com ambientes de exposição interno, para as cinco cidades, teriam a despassivação da armadura como um evento certo, e mais, que Brasília é a capital que apresenta a maior velocidade de carbonatação, isso se deve a média da umidade, que foi de $63,54 \%$. Os outros estados apresentaram umidade médias entre 70 e $78 \%$.

Dando continuidade na análise dos resultados, é possível observar por meio da Figura 5, que para os cenários simulados neste trabalho, e considerando a análise determinística, não ocorre a despassivação do aço para o ambiente externo desprotegido da chuva. Desta forma, para estes casos, as estruturas de concretos teriam sua vida útil de projeto garantida, apresentando a durabilidade requisitada ainda em projeto.

A seguir, na Figura 6, são apresentadas as probabilidades de os elementos de concreto atingirem o estado limite de despassivação durante o período que compreende a vida útil de projeto. A determinação das probabilidades foi realizada mediante simulação de Monte Carlo, já descrito neste trabalho. Ao todo foram geradas 50.000 amostras para a realização das simulações e cálculo de probabilidades.
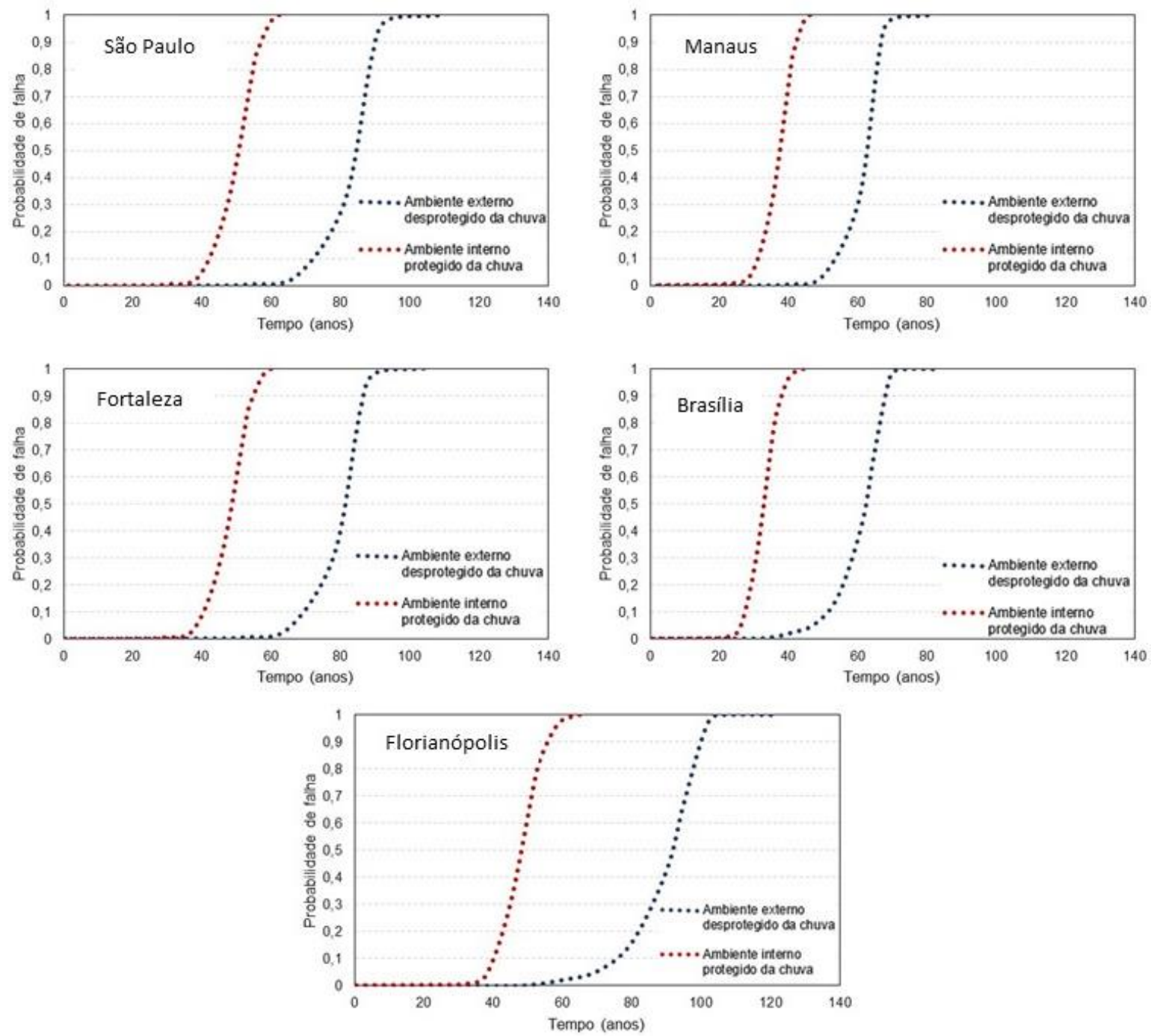

Figura 5: Probabilidade de os elementos atingirem o estado limite de serviço.

A partir dos resultados apresentados na Figura 6, verificou-se que a probabilidade de falha, é nula para os 20 primeiros anos de vida, independentemente da cidade e do ambiente de exposição. Observa-se também, que as probabilidades de falha aumentam rapidamente para as cidades analisadas, e que, as estruturas de concreto entram em despassivação antes de atingirem o fim da vida útil de projeto para todos os casos, com exceção, da viga produzida em São Paulo e em ambiente desprotegido da chuva. 
Na Figura 6 é possível observar ainda que, o ambiente de exposição interno protegido da chuva, apresenta condições mais agravante que o ambiente externo desprotegido, e que a depender da região em que a estrutura de concreto está inserida, a probabilidade de falha para o período de vida útil de projeto do elemento, é significativamente maior, demonstrando a importância de se conhecer corretamente o ambiente de exposição do concreto, definindo ainda em projeto as condições climáticas da região de construção, uma vez que é no meio ambiente onde são encontrados ao agentes deteriorantes.

\section{CONCLUSÕES}

Diante dos resultados obtidos com as análises determinísticas e probabilísticas, pode-se concluir que:

- Um cobrimento adequado garante não só um período maior para levar à despassivação da armadura como também para atingir o limite de abertura de fissuras;

- Diante das análises, observou-se que das quatro variáveis aleatórias utilizadas, a UR e o $\mathrm{f}_{\text {ck }}$, foram as que apresentaram maior relevância e sensibilidade frente à probabilidade de a viga atingir a despassivação durante sua vida útil de projeto;

- Quanto à análise da proteção da estrutura à chuva, observou-se que a viga, independentemente do local onde foi concebida, possui maior probabilidade de alcançar a despassivação quando situada em ambiente interno protegido da chuva, e mais, que essa probabilidade é significativamente maior que a da viga em ambiente externo desprotegido.

Assim, considerando os resultados obtidos neste trabalho, a durabilidade de estruturas de concreto armado deve ser associada a valores mais rigorosos de espessura de cobrimento, bem como concretos menos permeáveis e uma melhor análise do futuro ambiente de exposição.

Acredita-se, ainda, que a abordagem probabilística do problema da vida útil através da consideração da aleatoriedade das variáveis envolvidas, demonstrou-se adequada para uma determinação mais realística da despassivação do aço, fornecendo assim, informações necessárias e importantes para a criação de programas de inspeção e manutenção da estrutura no decorrer de sua vida.

\section{AGRADECIMENTOS}

Os autores agradecem ao Centro Universitário Estácio de Ribeirão Preto pelo fomento fornecido à pesquisa.

\section{REFERÊNCIAS}

ALMUSALLAM, A. A.; AL-GAHTANI, A. S.; AZIZ, A. R.; RASHEEDUZZAFAR, Effect of reinforcement corrosion on bond strength. Construction and Building Materials, v. 10, n. 2, p. 123-129, 1996.

ANDRADE, C., Manual para diagnóstico de obras deterioradas por corrosão de armaduras. Trad. De Antonio Carmona e Paulo Helene. São Paulo, Pini, 1992.

ASSOCIAÇÃO BRASILEIRA DE NORMAS TÉCNICAS. NBR 6118: Projeto de estruturas de concreto. Rio de Janeiro, 2014.

BECK, A. T. Confiabilidade e segurança das estruturas. São Paulo: Elsevier Campus, p. 448. 2019.

CARMONA, A. F.; MAREGA, A., Retrospectiva da patologia no Brasil: Estudo Estatístico, in: Jornadas em Español y Portugués sobre Estructuras y Materiales, CEDEX, IETcc, (1988), pp. 325-348, 1988.

CHANG, C. F.; CHEN, J. W., The experimental investigation of concrete carbonation depth, Cement and Concrete Research, V.36, pp. 1760-1767, 2006.

DAL MOLIN, D. C. C., Fissuras em estruturas de concreto armado: análise das manifestações típicas e levantamento de casos ocorridos no Estado do Rio Grande do Sul, Dissertação de Mestrado em Engenharia, Universidade Federal do Rio Grande do Sul, Porto Alegre, (1988). 
ELLINGWOOD, B. R.; FRANGOPOL, D. M., Introduction to the State of the Art Collection: Risk-Based Lifecycle Performance of Structural Systems, Journal of Structural Engineering, V.142, pp. 1, 2016.

FELIX, E. F.; BALABUCH, T. J. R.; CARRAZEDO, R.; POSSAN, E. Análise da vida útil de serviço de estruturas de concreto armado sujeitas à corrosão: uma abordagem mecânico-probabilística, in: Congresso Latinoamericano de Patologia de Construcción, CONPAT, Chiapas, México, 2019.

GEIKER, M. R.; POLDER, R. B., Experimental support for new electro active repair method for reinforced concrete, Materials and Corrosion, V.67, pp. 600-606, 2016.

HAMADA, M., Neutralization (carbonation) of concrete and corrosion of reinforcing steel. In: International Symposium on the Chemistry of Cement, 5, 1969. Tokyo. Proceedings... Tokyo, 1969, Part. III, v. II/4, p.343-369.

HELENE, P. R. L., Corrosão em Armaduras para Concreto Armado. São Paulo: IPT, PINI, 1986.

HELENE, P. R. L., Introdução. In: Ribeiro, D. V.(org). Corrosão e degradação em estruturas de concreto. Rio de Janeiro: Elsevier, 2018.

KARI, O. P.; PUTTONEN, J.; SKANTZ, E., Reactive transport modelling of long-term carbonation, Cement and Concrete Composites, v.52, pp. 42-53, 2014.

KÖLIÖ, A.; PAKKALA, T. A.; HOHTI, H.; LAUKKARINEN, A.; LAHDENSIVU,J.; MATTILA, J.; PENTTI, M., The corrosion rate in reinforced concrete facades exposed to outdoor environment, Materials and Structures, V.50, pp. 1$16,2017$.

LIBERATI, E. A. P.; LEONEL, E. D.; NOGUEIRA, C. G. Influence of the reinforcement corrosion on the bending moment capacity of reinforced concrete beams: a structural reliability approach. Ibracon Structures and Materials Journal. 7(3):379-413, 2014.

MEHTA, P. K.; MONTEIRO, P. J. M. Concreto. Microestrutura, propriedades e materiais. 4. ed. São Paulo: IBRACON, 2014.

NEVILLE, A. M., Propriedades do concreto, São Paulo: PINI, pp. 828, 1997.

NGUYEN, T. T. H.; BARY, B.; DE LARRARD, T., Coupled carbonation-rust formation damage modeling and simulation of steel corrosion in 3D mesoscale reinforced concrete. Cement and Concrete Research, v. 74, p. 95-107, 2015.

PAPADAKIS, V. G.; VAYENAS, C. G.; FARDIS, M. N., Fundamental Modeling and Experimental Investigation of Concrete Carbonation. ACI Materials Journal, v.88, n.4, July-Auguste, 1991.

POSSAN, E. Modelagem da carbonatação e previsão de vida útil de estruturas de concreto em meio urbano, Tese de Doutorado em Engenharia, Programa de Pós-Graduação em Engenharia Civil, Universidade Federal do Rio Grande do Sul, Porto Alegre, 2010.

SMOLCZYK, H. G., Written Discussion. In: The International Symposium on the Chemistry of Cement, V. Tokyo, 1969. Proceedings... Tokyo, 1969, Part III, v. II/4, p.369-384.

TUUTTI, K., Corrosion of Steel in Concrete. Swedish Cement and Concrete Research Institute. Stockholm, Swedish, 1982. 30p. 